\title{
Generalized dilaton-axion models of inflation, de Sitter vacua and spontaneous SUSY breaking in supergravity
}

\author{
Yermek Aldabergenov ${ }^{1,2, a}$, Auttakit Chatrabhuti ${ }^{1, b}$, Sergei V. Ketov ${ }^{3,4,5, \mathrm{c}}$ \\ ${ }^{1}$ Department of Physics, Faculty of Science, Chulalongkorn University, Thanon Phayathai, Pathumwan, Bangkok 10330, Thailand \\ ${ }^{2}$ Institute for Experimental and Theoretical Physics, Al-Farabi Kazakh National University, 71 Al-Farabi Avenue, 050040 Almaty, Kazakhstan \\ ${ }^{3}$ Department of Physics, Tokyo Metropolitan University, Minami-ohsawa 1-1, Hachioji, Tokyo 192-0397, Japan \\ ${ }^{4}$ Research School for High Energy Physics, Tomsk Polytechnic University, 30 Lenin Avenue, Tomsk 634050, Russian Federation \\ ${ }^{5}$ Kavli Institute for the Physics and Mathematics of the Universe (IPMU), The University of Tokyo, Chiba 277-8568, Japan
}

Received: 5 August 2019 / Accepted: 13 August 2019 / Published online: 24 August 2019

(C) The Author(s) 2019

\begin{abstract}
We propose the unified models of cosmological inflation, spontaneous SUSY breaking, and the dark energy (de Sitter vacuum) in $N=1$ supergravity with the dilatonaxion chiral superfield $T$ in the presence of an $N=1 \mathrm{vec}-$ tor multiplet with the alternative Fayet-Iliopoulos term. By using the Kähler potential as $K=-\alpha \log (T+\bar{T})$ and the superpotential as a sum of a constant and a linear term, we find that viable inflation is possible for $3 \leq \alpha \leq \alpha_{\max }$ where $\alpha_{\max } \approx 7.235$. Observations of the amplitude of primordial scalar perturbations fix the SUSY breaking scale in our models as high as $10^{13} \mathrm{GeV}$. In the case of $\alpha>3$ the axion gets the tree-level (non-tachyonic) mass comparable to the inflaton mass.
\end{abstract}

\section{Introduction}

Supergravity is well motivated as the possible theoretical interface between (a) high-energy physics (well) beyond the Standard Model (SM) of elementary particles, (b) gravity beyond the Concordance $(\Lambda \mathrm{CDM})$ Cosmological Model, and (c) string theory as the theory of quantum gravity whose lowenergy effective action is described by supergravity. A phenomenological description of high energy particle physics and cosmology in supersymmetry (SUSY) and supergravity is known to be non-trivial, though many viable models exist, see e.g., the reviews [1-4] and the references therein. No signs of SUSY at the Large Hadron Collider (LHC) may hint towards a high scale of SUSY phenomena. At such scales (indirect) cosmological probes of SUSY prevail over (direct) experimental probes at particle colliders. The early Universe

\footnotetext{
a e-mail: yermek.a@chula.ac.th

be-mail: auttakit.c@chula.ac.th

c e-mail: ketov@tmu.ac.jp
}

is, therefore, the natural place for physical applications of supergravity.

A simultaneous description of cosmological inflation and dark energy (as the positive cosmological constant) in supergravity is another challenge due to the huge difference in the relevant scales and the need of (spontaneous) SUSY breaking. The standard approach in supergravity is based on the use of chiral $N=1$ superfields in four spacetime dimensions with the input given by a Kähler potential $K$ and a superpotential $W$. Then the scalar potential and the kinetic terms of the scalar field components are uniquely defined, and the phenomenological model building amounts to choosing both $K$ and $W$ in order to achieve a viable single-field inflation consistent with the Cosmic Microwave Background (CMB) observations and a de Sitter (dS) vacuum after inflation. There are several problems with that approach. First, the input given by $K$ and $W$ allows infinitely many choices. Second, it always leads to the multi-scalar framework so that one has to choose the inflaton direction in the field space and suppress the non-inflaton scalars during inflation in order to prevent spoiling of the inflaton slow roll and get enough number of e-foldings. Third, after inflation one has to get the hierarchy between the (high) SUSY breaking scale allowing large masses for the superpartners of the SM particles and the (low) dark energy scale given by the cosmological constant. Getting that hierarchy may require two different mechanisms of spontaneous SUSY breaking.

It is possible to reduce (and minimize) the number of scalars in the inflationary models by employing a massive (irreducible) $N=1$ vector multiplet as the inflaton supermultiplet, instead of a chiral one [5,6]. The massive vector multiplet has only one (real) physical scalar that can be identified with inflaton, while its fermionic superpartner can be identified with goldstino in the minimalistic setup for 
inflation in supergraviity (cf. Refs. [7,8]). To avoid SUSY restoration after inflation in a Minkowski vacuum (it was the drawback of the first supergravity models with inflaton in a vector multiplet), one may either add the hidden sector described by a chiral (Polonyi) superfield as in Refs. [911] or introduce the alternative (new) Fayet-Iliopoulos (FI) terms as in Refs. $[12,13] .{ }^{1}$ Moreover, one can also combine both approaches and derive the supergravity-based inflationary models with inflaton in a massive vector multiplet in the presence of the FI term, with both F-type and D-type SUSY breaking needed for the hierarchy of scales $[16,17]$. In all those cases, the canonical Kähler potential and a linear superpotential for Polonyi superfield were chosen, like the original Polonyi model [18].

Another approach is based on the use of the "dilatonaxion" superfield $T$ by replacing the canonical (free) Kähler potential by the generalized "no-scale" one as follows [19]:

$K=-\alpha \log (T+\bar{T})$,

The corresponding $N=1$ non-linear sigma-model has the $S L(2 ; \mathbb{R}) / S O(2)$ (or $S U(1,1 ; \mathbb{C}) / U(1))$ tangent space of Kähler curvature $R_{K}=2 / \alpha$, and is of particular interest for particle phenomenology because such Kähler potential in the case of $\alpha=3$ arises in generic heterotic string compactifications and allows for the realistic particle model building [19-22]. Then $T$ can be identified with the volume modulus of the compactified manifold in heterotic string theory. It is remarkable that the same Kähler potential with $\alpha=3$ also arises in the modified $F(R)$ supergravity after its dualization [23-25].

It is, however, also known that the case of $\alpha=3$ in Eq. (1) with just a single chiral superfield is not viable for cosmological applications because it does not allow stable dS vacua and cannot be used for realizing Starobinsky inflation [26] with any choice of the superpotential $W$ [3,27-30], although there are single field models with generalized $\alpha$ ( $\alpha$-attractors) leading to a supersymmetric Minkowski vacuum [31-33].

The "no-scale" supergravity was successfully used for describing inflation in Refs. [27-30,34,35] with the help of at least two chiral superfields and the Kähler potential

$K=-3 \log \left(T+\bar{T}-\sum_{i=1}^{p-1}\left|\Phi^{i}\right|^{2} / 3\right)$,

where $T$ is the volume modulus, and $\Phi^{i}$ are the matter chiral superfields parametrizing the non-linear sigma-model tangent space $S U(p, 1 ; \mathbb{C}) / S U(p) \times U(1)$, with the suitable superpotential.

\footnotetext{
1 The alternative FI terms without gauging the R-symmetry were introduced in Refs. $[14,15]$.
}

In this paper we use a single "dilaton-axion" chiral superfield $T$ with the Kähler potential (1) but introduce a single vector multiplet in addition. We demonstrate that it leads to the viable set of cosmological models describing inflation, dS vacua and spontaneous SUSY breaking.

We recall that the original Starobinsky model of inflation [26] is based on the modified $\left(R+R^{2}\right)$ gravity, while its extension in the new-minimal formulation of supergravity has the dual description in terms of the standard supergravity coupled to a massive vector multiplet or, equivalently, a massless vector multiplet and a Stückelberg chiral multiplet with the Kähler potential [6]

$K=-3 \log (T+\bar{T})+3(T+\bar{T})$.

The last term can be identified with the FI term of the gauged R-symmetry (in a non-R-symmetric frame), because the Dterm of this model results in the Starobinsky potential $[5,6$, 36].

The authors of Ref. [37] studied even more general models of a single chiral multiplet and an abelian vector multiplet with the gauged R-symmetry and the Kähler potential having two parameters $\alpha$ and $\beta$,

$K=-\alpha \log (T+\bar{T})+\beta(T+\bar{T})$,

and found that slow-roll inflation consistent with observations is only possible for $\alpha=1,2$ after adding some nonperturbative corrections. SUSY is spontaneously broken after inflation in those models, with the gravitino mass in the $\mathrm{TeV}$ range.

In this paper we find that a non-vanishing D-term allows us to introduce the new inflationary models based on the Kähler potentials having the form (1) with a single chiral superfield and a single vector superfield. The other examples of the Dterm based on the alternative FI terms $[14,15]$ can be found in Refs. [13,38-41]. Those FI terms provide a tunable positive cosmological constant or dS uplifting of the vacuum after inflation $[12,16,17,42]$. Our inflationary models in this paper have the Kähler potential (1) and the Polonyi-type linear superpotential (without gauging the shift symmetry of $K$ ) leading to the spontaneous F-type SUSY breaking. In addition, the simplest alternative FI term leads to another D-type SUSY breaking and uplifts an Anti-dS (AdS) minimum of the F-term scalar potential to a dS minimum.

The paper is organized as follows. Our setup is given in Sect. 2. In Sect. 3 we study vacua and SUSY breaking. In Sect. 4 we study inflation in our framework and analyze in detail the models with integer $\alpha$. In particular, we derive the explicit values of the dilaton and axion masses and the SUSY breaking parameters by fixing the inflationary observables with the CMB observational data. We conclude in Sect. 5. The basic formulae about the standard $N=1$ supergravity 
and the alternative FI term are given in Appendix. We set the reduced Planck mass as $M_{\mathrm{Pl}}=\kappa^{-1}=1$ unless otherwise stated.

\section{The setup}

Let us consider the following Kähler potential and the superpotential:

$K=-\alpha \log (T+\bar{T})$,

$W=\lambda+\mu T$,

where $\alpha$ is a positive real constant, $\lambda$ and $\mu$ are complex parameters. The $T$ is parametrized as

$T=e^{-\sqrt{\frac{2}{\alpha}} \phi}+i t$

in terms of the canonical inflaton $\phi$ and its axionic partner $t$. The F-term scalar potential reads

$$
\begin{aligned}
V_{F}= & e^{K}\left[K^{T \bar{T}}\left(W_{T}+K_{T} W\right)\left(\bar{W}_{\bar{T}}+K_{\bar{T}} \bar{W}\right)-3|W|^{2}\right] \\
= & \frac{\alpha-3}{2^{\alpha}}\left(|\lambda|^{2}+\omega_{2} t+|\mu|^{2} t^{2}\right) e^{\sqrt{2 \alpha} \phi} \\
& +\frac{(\alpha-5) \omega_{1}}{2^{\alpha}} e^{(\alpha-1) \sqrt{\frac{2}{\alpha}} \phi} \\
& +\frac{\left(\alpha^{2}-7 \alpha+4\right)|\mu|^{2}}{2^{\alpha} \alpha} e^{(\alpha-2) \sqrt{\frac{2}{\alpha}} \phi},
\end{aligned}
$$

where we have used the parametrization (7) and the notation

$$
\begin{aligned}
& \omega_{1} \equiv \bar{\lambda} \mu+\lambda \bar{\mu}=2 \lambda_{R} \mu_{R}+2 \lambda_{I} \mu_{I}, \\
& \omega_{2} \equiv i(\bar{\lambda} \mu-\lambda \bar{\mu})=2 \lambda_{I} \mu_{R}-2 \lambda_{R} \mu_{I} .
\end{aligned}
$$

The subscripts $R, I$ stand for the real and imaginary parts. It is convenient to trade the complex parameter $\lambda$ for the two real ones, $\omega_{1}$ and $\omega_{2}$ defined above.

A generic vacuum of the F-term potential (8) is AdS. However, after introducing an abelian vector multiplet with the simplest alternative FI term $[14,15]$ and eliminating the auxiliary field $(D)$ of the vector multiplet, one gets a positive contribution

$V_{D}=\frac{g^{2} \xi^{2}}{2}$

to the cosmological constant, where $g$ is the gauge coupling, and $\xi$ is the real FI constant. ${ }^{2}$ More details about the alternative FI term and the bosonic action of the standard $N=1$ supergravity can be found in Appendix.

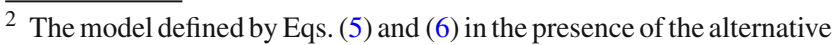
FI term and the linear gauge kinetic function $f(g)=T$ leads to the vanishing scalar potential [see Eqs. (2) and (8)] when $\alpha=3$ and $\omega_{1}=0$ [43], which is the defining property of the "no-scale" supergravity.

\section{Vacua and SUSY breaking}

Let us analyze minima of the scalar potential (8). The vacuum equations for the critical points and the critical value $V_{0}$ of the potential read

$$
\begin{aligned}
& V_{0}=A x^{\alpha}+B x^{\alpha-1}+C x^{\alpha-2}+\frac{g^{2} \xi^{2}}{2} \\
& V_{x}=\alpha A x^{\alpha-1}+(\alpha-1) B x^{\alpha-2}+(\alpha-2) C x^{\alpha-3}=0 \\
& V_{t}=\frac{\alpha-3}{2^{\alpha}}\left(\omega_{2}+2|\mu|^{2} t_{0}\right) x^{\alpha}=0
\end{aligned}
$$

where we have used the notation

$x \equiv e^{\sqrt{\frac{2}{\alpha}} \phi_{0}} \quad$ with $\quad \phi_{0} \equiv\langle\phi\rangle$,

$A \equiv \frac{\alpha-3}{2^{\alpha}}\left(|\lambda|^{2}+\omega_{2} t_{0}+|\mu|^{2} t_{0}^{2}\right)$,

$B \equiv \frac{(\alpha-5) \omega_{1}}{2^{\alpha}}, \quad C \equiv \frac{\left(\alpha^{2}-7 \alpha+4\right)|\mu|^{2}}{2^{\alpha} \alpha}$,

and $V_{x} \equiv \partial V / \partial x$.

The special value $\alpha=3$ yields the identically vanishing $A$ and $V_{t}$, thus making the potential $t$-independent. In the next Section we consider separately the case of $\alpha=3$ and then turn to $\alpha \neq 3$. When $\alpha \neq 3$, we can use the solution to Eq. (14) as $t_{0}=-\omega_{2} /\left(2|\mu|^{2}\right)$ and rewrite $A$ as

$A=\frac{\alpha-3}{2^{\alpha}}\left(|\lambda|^{2}-\frac{\omega_{2}^{2}}{4|\mu|^{2}}\right)=\frac{(\alpha-3) \omega_{1}^{2}}{2^{\alpha+2}|\mu|^{2}}$,

where in the last equation we have used the definitions (9) and (10). Since $\omega_{1}$ is real, $A$ becomes negative when $\alpha<3$. Given negative $A$, the potential (12) is unbounded from below because $A$ multiplies the highest power of $x$ (for $\alpha<3$ the potential also becomes unstable in the $t$-direction). Therefore, we restrict ourselves to $\alpha \geq 3$ in what follows.

\subsection{The case $\alpha=3$}

Given $\alpha=3$, the scalar potential takes the simple form

$V=-\frac{\omega_{1}}{4} e^{\sqrt{\frac{8}{3}} \phi}-\frac{|\mu|^{2}}{3} e^{\sqrt{\frac{2}{3}} \phi}+\frac{g^{2} \xi^{2}}{2}$,

and has a minimum at

$$
\begin{aligned}
\phi_{0} & =\sqrt{\frac{3}{2}} \log \left(-\frac{2|\mu|^{2}}{3 \omega_{1}}\right), \\
V_{0} & =\frac{g^{2} \xi^{2}}{2}+\frac{|\mu|^{4}}{9 \omega_{1}} .
\end{aligned}
$$


The minimum exists only if $\omega_{1}<0$. The minimum is AdS, Minkowski, or $\mathrm{dS}$, depending on the following relations:

$$
\begin{aligned}
& g^{2} \xi^{2}<\frac{2|\mu|^{4}}{9\left|\omega_{1}\right|} \longrightarrow \text { AdS }, \\
& g^{2} \xi^{2}=\frac{2|\mu|^{4}}{9\left|\omega_{1}\right|} \longrightarrow \text { Minkowski, } \\
& g^{2} \xi^{2}>\frac{2|\mu|^{4}}{9\left|\omega_{1}\right|} \longrightarrow \mathrm{dS} .
\end{aligned}
$$

Hence, by fine-tuning the parameters we can obtain a small positive cosmological constant $V_{0}$ for the realistic phenomenology.

Defining $\varphi \equiv \phi-\phi_{0}$ as excitation of the inflaton around its Vacuum Expectation Value (VEV), the potential (17) can be brought to the form [after using Eq. (19) to eliminate $g \xi$ in terms of $V_{0}$ ]

$V=V_{0}+\frac{|\mu|^{4}}{9\left|\omega_{1}\right|}\left(e^{\sqrt{\frac{2}{3} \varphi}}-1\right)^{2}$

that gives the realization of the Starobinsky inflationary model (with the cosmological constant) in our framework. The potential is $t$-flat.

SUSY is spontaneously broken by the constant nonvanishing D-term, $D=\langle D\rangle=g \xi$, while $\left\langle F_{T}\right\rangle$ and the gravitino mass are given by

$$
\begin{aligned}
\left\langle F_{T}\right\rangle & =\left\langle-e^{K / 2} K^{T \bar{T}}\left(\bar{W}_{\bar{T}}+K_{\bar{T}} \bar{W}\right)\right\rangle \\
& =-\frac{i \operatorname{sgn}(\mu)}{\sqrt{12\left|\omega_{1}\right|}}\left(\omega_{2}+2|\mu|^{2} t_{0}\right), \\
m_{3 / 2}^{2} & =\left\langle e^{K}|W|^{2}\right\rangle=\left|-2 \omega_{1}+i\left(\omega_{2}+2|\mu|^{2} t_{0}\right)\right|^{2} \frac{|\mu|^{4}}{108\left|\omega_{1}\right|^{3}} .
\end{aligned}
$$

Though $\left\langle F_{T}\right\rangle$ is arbitrary (and may even vanish), the gravitino mass is bounded from below,

$m_{3 / 2}^{2} \geq \frac{|\mu|^{4}}{27\left|\omega_{1}\right|}$

The mass of the inflaton is

$m_{\varphi}^{2}=\frac{4|\mu|^{4}}{27\left|\omega_{1}\right|}$

so that we have the relation $2 m_{3 / 2} \geq m_{\varphi}$.

The bosonic sector also includes a massless axion $t$ and a massless vector. The vector can be made massive via (additional) super-Higgs effect. A massless scalar is phenomenologically problematic, but the mass of $t$ may be generated either by quantum corrections when $\alpha=3$ (as is usually assumed in the "no-scale" supergravity models), or already at the tree level when $\alpha>3$, as we are going to show in the next Subsection.

3.2 The case $\alpha>3$ : vacuum solutions

If the axion field is fixed at its VEV, $t_{0}=-\omega_{2} /\left(2|\mu|^{2}\right)$, we can rewrite the scalar potential (8) and (2) for $\alpha>3$ as

$$
\begin{aligned}
V= & \frac{(\alpha-3) \omega_{1}^{2}}{2^{\alpha+2}|\mu|^{2}} e^{\sqrt{2 \alpha} \phi}+\frac{(\alpha-5) \omega_{1}}{2^{\alpha}} e^{(\alpha-1) \sqrt{\frac{2}{\alpha}} \phi} \\
& +\frac{\left(\alpha^{2}-7 \alpha+4\right)|\mu|^{2}}{2^{\alpha} \alpha} e^{(\alpha-2) \sqrt{\frac{2}{\alpha}} \phi}+\frac{1}{2} g^{2} \xi^{2},
\end{aligned}
$$

assuming $\omega_{1} \neq 0$. The vacuum equations (12) and (13) then take the form

$$
\begin{aligned}
V_{0}= & \frac{(\alpha-3) \omega_{1}^{2}}{2^{\alpha+2}|\mu|^{2}} x^{\alpha}+\frac{(\alpha-5) \omega_{1}}{2^{\alpha}} x^{\alpha-1} \\
& +\frac{\left(\alpha^{2}-7 \alpha+4\right)|\mu|^{2}}{2^{\alpha} \alpha} x^{\alpha-2}+\frac{1}{2} g^{2} \xi^{2}, \\
V_{x}= & \frac{\alpha(\alpha-3) \omega_{1}^{2}}{2^{\alpha+2}|\mu|^{2}} x^{\alpha-1}+\frac{(\alpha-1)(\alpha-5) \omega_{1}}{2^{\alpha}} x^{\alpha-2} \\
& +\frac{(\alpha-2)\left(\alpha^{2}-7 \alpha+4\right)|\mu|^{2}}{2^{\alpha} \alpha} x^{\alpha-3}=0,
\end{aligned}
$$

where $x \equiv e^{\phi_{0} / \sqrt{2}}$ as before. Equation (30) has two solutions,

$x_{+}=\frac{2\left(-\alpha^{2}+7 \alpha-4\right)|\mu|^{2}}{\alpha(\alpha-3) \omega_{1}}, \quad x_{-}=\frac{2(2-\alpha)|\mu|^{2}}{\alpha \omega_{1}}$

that we parametrize as

$x_{ \pm}=\gamma_{ \pm} \frac{|\mu|^{2}}{\omega_{1}}\left\{\begin{array}{l}\gamma_{+} \equiv \frac{2\left(-\alpha^{2}+7 \alpha-4\right)}{\alpha(\alpha-3)} \\ \gamma_{-} \equiv \frac{2(2-\alpha)}{\alpha}\end{array}\right.$

The positivity of $x$ requires $\gamma / \omega_{1}$ to be positive. Since we have $\alpha>3$, the $\gamma_{-}$is always negative, while the sign of $\gamma_{+}$ depends on the choice of $\alpha$. More specifically, we find ${ }^{3}$

$3<\alpha<\frac{1}{2}(7+\sqrt{33}) \longrightarrow \gamma_{+}>0$

Hence, if $\omega_{1}>0$, the $x_{+}$should be used as the vacuum solution in this parameter region. On the other hand,

$\alpha=\frac{1}{2}(7+\sqrt{33}) \longrightarrow \gamma_{+}=0$

that invalidates the $x_{+}$as a stable solution for the given value of $\alpha$. Thus $\omega_{1}$ must be negative and $x_{-}$should be used as the

${ }^{3} \alpha=\frac{1}{2}(7+\sqrt{33})$ is one of the two roots of the polynomial $\alpha^{2}-7 \alpha+4$ that yields $\gamma_{+}=x_{+}=0$. Another root is $\alpha=\frac{1}{2}(7-\sqrt{33})<3$ so that it is excluded from the analysis. 


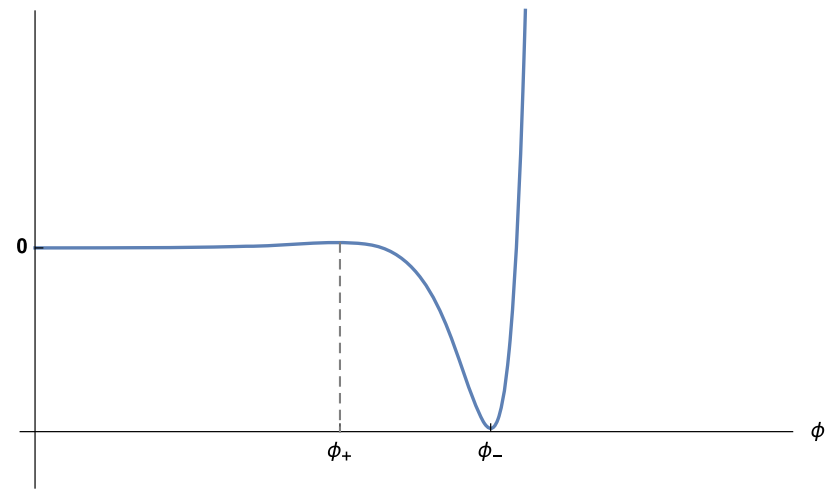

Fig. 1 The form of the scalar potential (28) for $\alpha>\frac{1}{2}(7+\sqrt{33})$, $\xi=0$, and negative $\omega_{1}$. The $\phi_{ \pm}$are defined as $x_{ \pm}=e^{\phi_{ \pm} / \sqrt{2}}$, where $\phi_{-}=\phi_{0}$ is the VEV of $\phi$

minimum. Moreover,

$\alpha>\frac{1}{2}(7+\sqrt{33}) \longrightarrow \gamma_{+}<0$.

This means that $\omega_{1}$ should be negative. In this case, both $x_{+}$ and $x_{-}$(i.e. $\phi_{+}$and $\phi_{-}$) are the valid stationary points and, in fact, the extrema - not inflection points - because

$\left.\frac{\partial^{2} V(\phi)}{\partial \phi^{2}}\right|_{\phi=\phi_{ \pm}} \neq 0$

where $V(\phi)$ is given by Eq. (28). Setting $\xi=0$ and using $\omega_{1}=-\left|\omega_{1}\right|$, we get

$\left.V_{0}\right|_{\phi=\phi_{+}}=\frac{|\mu|^{2(\alpha-1)}}{2^{\alpha+2} \alpha\left|\omega_{1}\right|^{\alpha-2}}\left(\frac{\alpha^{2}-7 \alpha+4}{\alpha(\alpha-3)}\right)^{\alpha-1}>0$,

$\left.V_{0}\right|_{\phi=\phi_{-}}=-\frac{3|\mu|^{2(\alpha-1)}}{2^{\alpha+2}\left|\omega_{1}\right|^{\alpha-2}}\left(\frac{1-\frac{2}{\alpha}}{(\alpha-2)^{2}}\right)<0$,

for $\alpha>(7+\sqrt{33}) / 2$. It means that $\phi_{+}$is a local maximum, while $\phi_{-}$is a global minimum. The general form of the potential is shown in Fig. 1. The existence of the local maximum at $\phi_{+}$means that the potential is of the hilltoptype and, therefore, we should consider inflation in the cases $3 \leq \alpha \leq(7+\sqrt{33}) / 2$ and $\alpha>(7+\sqrt{33}) / 2$ separately. Since the value of $\alpha=(7+\sqrt{33}) / 2$ is special, we introduce the notation

$\alpha_{*} \equiv \frac{1}{2}(7+\sqrt{33}) \approx 6.37$

It is noteworthy that the choice of $\alpha=5$ leads to $\gamma_{+}=$ $-\gamma_{-}=6 / 5$, so that the scalar potentials in the cases $\omega_{1}>0$ and $\omega_{1}<0$ exactly coincide.
3.3 The case $\alpha>3$ : SUSY breaking and scalar masses

In the case of $3<\alpha \leq \alpha_{*}$ we use again the axion VEV, $t_{0}=-\omega_{2} /\left(2|\mu|^{2}\right)$, and the general solution $x=\gamma|\mu|^{2} / \omega_{1}$ to find $F_{T}$ and the gravitino mass at the minimum,

$$
\begin{aligned}
& \left\langle F_{T}\right\rangle=\frac{(\gamma \alpha+2 \alpha-4)|\mu|^{2-\alpha}}{2^{\alpha / 2} \alpha \mu}\left(\frac{\gamma}{\omega_{1}}\right)^{\frac{\alpha}{2}-2}, \\
& m_{3 / 2}^{2}=\frac{(\gamma+2)^{2}|\mu|^{2(\alpha-1)}}{4 \cdot 2^{\alpha}}\left(\frac{\gamma}{\omega_{1}}\right)^{\alpha-2} .
\end{aligned}
$$

Substituting the two solutions from Eq. (32) yields

$$
\begin{aligned}
& \left.\left\langle F_{T}\right\rangle\right|_{x_{+}}=\frac{(\alpha+1)|\mu|^{\alpha-2}}{\alpha^{\frac{\alpha}{2}-1}(\alpha-3) \mu}\left[\frac{\alpha^{2}-7 \alpha+4}{(3-\alpha) \omega_{1}}\right]^{\frac{\alpha}{2}-2}, \\
& \left.\left\langle F_{T}\right\rangle\right|_{x_{-}}=0
\end{aligned}
$$

while the gravitino mass in both cases $\left(x_{+}\right.$and $\left.x_{-}\right)$is nonvanishing. Now recall that for $3<\alpha<\alpha_{*}$ the vacuum solution is $x_{+}$if $\omega_{1}$ is positive, and $x_{-}$if $\omega_{1}$ is negative, while for $\alpha \geq \alpha_{*}$ only $x_{-}$can be a stable vacuum solution and this requires a negative $\omega_{1}$. Thus, we conclude that if $3<\alpha<\alpha_{*}$, a positive $\omega_{1}$ leads to the mixed F- and D-term SUSY breaking, while a negative $\omega_{1}$ leads to the pure D-term SUSY breaking. If $\alpha \geq \alpha_{*}$, only the pure D-term breaking is possible (we exclude runaway solutions).

As for the mass of the axion $t$, we first get

$m_{t}^{2}=\frac{(\alpha-3)|\mu|^{2}}{2^{\alpha-1}} e^{\sqrt{2 \alpha} \phi_{0}}$

However, the $t$ is not canonical at the $\phi$-minimum because the $\phi_{0}$ is non-vanishing and

$e^{-1} \mathcal{L}_{\text {kin }}\left(t, \phi_{0}\right)=-\frac{\alpha}{4} e^{\sqrt{\frac{8}{\alpha}} \phi_{0}}(\partial t)^{2}$

as can be seen from Eq. (71) in Appendix. Though it is impossible to canonically normalize the kinetic term of $t$ for all values of $\phi$, it is certainly possible at the reference point $\phi_{0}$ by the rescaling

$t=\sqrt{\frac{2}{\alpha}} e^{-\sqrt{\frac{2}{\alpha}} \phi_{0}} t^{\prime}$

where $t^{\prime}$ is the "canonical" axion. Its mass squared is then given by

$$
\begin{aligned}
m_{t^{\prime}}^{2} & =\frac{(\alpha-3)|\mu|^{2}}{2^{\alpha-2} \alpha} e^{(\alpha-2) \sqrt{\frac{2}{\alpha}} \phi_{0}} \\
& =\frac{(\alpha-3)|\mu|^{2(\alpha-1)}}{2^{\alpha-2} \alpha}\left(\frac{\gamma}{\omega_{1}}\right)^{\alpha-2},
\end{aligned}
$$


Fig. 2 The mass ratios $\Delta_{ \pm}$and $\Gamma_{ \pm}$evaluated at

$3<\alpha<\alpha_{*} \approx 6.37$. In the plot (a) the dashed lines denote the points on the $\alpha$-axis where $\Delta_{+}=1$ and $\Gamma_{+}=1$, whose values are (from the left to the right) approximately $3.8,5.27$, and 5.37
$-\Delta_{+}-\Gamma_{+}$

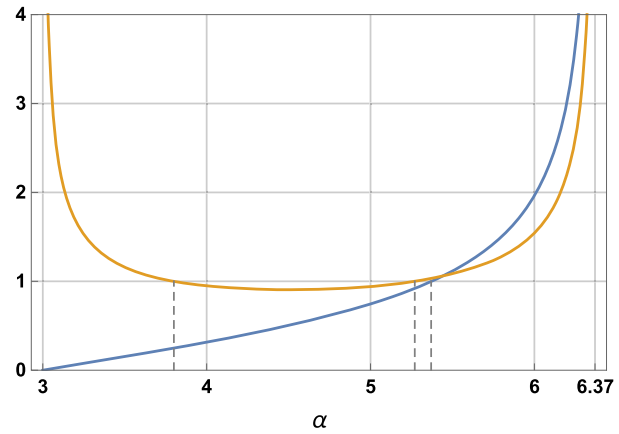

(a) $\Delta_{+}$(blue curve) and $\Gamma_{+}$(orange curve).
$-\Delta_{-}-\Gamma_{-}$

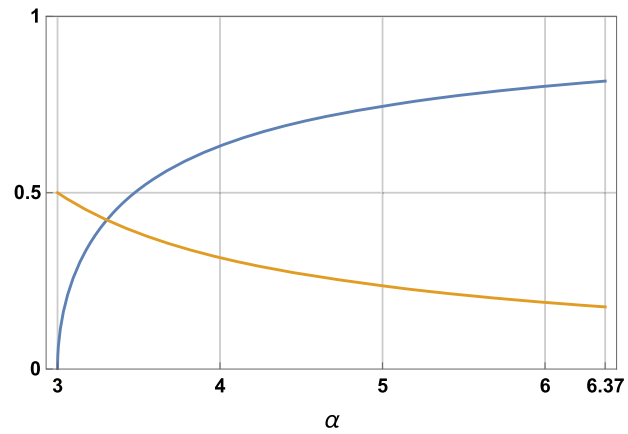

(b) $\Delta_{-}$(blue curve) and $\Gamma_{-}$(orange curve). where we have used the general vacuum solution $e^{\sqrt{\frac{2}{\alpha}} \phi_{0}} \equiv$ $x=\gamma|\mu|^{2} / \omega_{1}$.

The inflaton mass can be read off from Eq. (28) after using $\varphi=\phi-\phi_{0}$ and substituting the general $x$ solution (32). We find

$$
\begin{aligned}
m_{\varphi}^{2}= & 2\left(\frac{\gamma}{2}\right)^{\alpha} \\
& \times\left[\frac{\alpha(\alpha-3)}{4}+\frac{(\alpha-5)(\alpha-1)^{2}}{\alpha \gamma}\right. \\
& \left.+\frac{\left(\alpha^{2}-7 \alpha+4\right)(\alpha-2)^{2}}{\alpha^{2} \gamma^{2}}\right] \frac{|\mu|^{2(\alpha-1)}}{\omega_{1}^{\alpha-2}} .
\end{aligned}
$$

It is convenient to define the mass ratios

$$
\left.\Delta_{ \pm} \equiv \frac{m_{t^{\prime}}}{m_{\varphi}}\right|_{\gamma=\gamma_{ \pm}},\left.\quad \Gamma_{ \pm} \equiv \frac{m_{3 / 2}}{m_{\varphi}}\right|_{\gamma=\gamma_{ \pm}},
$$

where the $\gamma_{ \pm}$are defined in Eq. (32). The parameters $\mu$ and $\omega_{1}$ cancel out in $\Delta$ and $\Gamma$ that can be readily plotted as the functions of $\alpha$.

In Fig. 2 we plot the mass ratios for $3<\alpha<\alpha_{*}$. Figure $2 \mathrm{a}$ shows that with $\gamma_{+}$corresponding to a positive $\omega_{1}$ axion is lighter than inflaton if $\alpha<(5+\sqrt{33}) / 2 \approx 5.37$, whereas beyond this point axion becomes heavier. Gravitino (with $\gamma_{+}$) is slightly lighter than inflaton in the range $3.8 \lesssim \alpha \lesssim 5.27$, whereas outside this range gravitino becomes heavier. ${ }^{4}$

In the case of $\gamma_{-}$(see Fig. 2b), i.e. a negative $\omega_{1}$, both axion and gravitino are lighter than inflaton. As we already showed, $x_{-}$is the global minimum of the potential even when $\alpha>\alpha_{*}$, so that the mass ratios $\Delta_{-}$and $\Gamma_{-}$can be extrapolated for large values of $\alpha$ as

$\lim _{\alpha \rightarrow \infty} \Delta_{-}=1, \quad \lim _{\alpha \rightarrow \infty} \Gamma_{-}=0$

i.e. the axion mass approaches the inflaton mass, while the gravitino mass slowly vanishes for large $\alpha$.

4 The point $\alpha=(5+\sqrt{33}) / 2 \approx 5.37$ can be found by solving $\Delta_{+}=1$ that yields a quadratic equation for $\alpha$, while the points $\alpha \approx 3.8$ and $\alpha \approx 5.27$ are found numerically by solving a quartic equation coming from $\Gamma_{+}=1$.

\section{Inflation}

In order to study inflation, let us restore the gravitational constant $\kappa \equiv \sqrt{8 \pi G}=M_{P}^{-1}$. We choose the Kähler potential and the chiral field $T$ to be dimensionless, whereas the superpotential has the mass dimension three, $[W]=M^{3}$. It follows that $[\lambda]=[\mu]=M^{3}$ and $\left[\omega_{1}\right]=M^{6}$, where $[\ldots]$ stands for the mass dimension of the corresponding quantity. We also set $[g \xi]=M^{0}$ and $[\phi]=[\varphi]=M$.

It is convenient to express the FI constant $g \xi$ in terms of the cosmological constant $V_{0}$ by using Eq. (29) and the general $x$-solution (32). Restoring $\kappa$ results in the potential

$$
\begin{aligned}
V= & V_{0}+\kappa^{2}\left(\frac{\gamma}{2}\right)^{\alpha} \frac{|\mu|^{2(\alpha-1)}}{\omega_{1}^{\alpha-2}} \\
& \times\left[\frac{\alpha-3}{4} e^{\sqrt{2 \alpha} \kappa \varphi}+\frac{\alpha-5}{\gamma} e^{(\alpha-1) \sqrt{\frac{2}{\alpha}} \kappa \varphi}\right. \\
& +\frac{\alpha^{2}-7 \alpha+4}{\alpha \gamma^{2}} e^{(\alpha-2) \sqrt{\frac{2}{\alpha}} \kappa \varphi} \\
& \left.-\frac{\alpha(\gamma+2)^{2}}{4 \gamma^{2}}+\frac{(\gamma+2)(3 \gamma+14)}{4 \gamma^{2}}-\frac{4}{\alpha \gamma^{2}}\right] .
\end{aligned}
$$

In what follows we neglect the cosmological constant $V_{0}$.

We use the standard definitions of the slow-roll parameters,

$\epsilon \equiv \frac{1}{2 \kappa^{2}}\left(\frac{V^{\prime}(\varphi)}{V(\varphi)}\right)^{2}, \quad \eta \equiv \frac{1}{\kappa^{2}} \frac{V^{\prime \prime}(\varphi)}{V(\varphi)}$

Inflation ends when $\epsilon=1$ that translates into the value of the inflaton field at the end of inflation, $\varphi_{f}$. The scalar spectral index and the tensor-to-scalar ratio are related to the slow-roll parameters as

$n_{s}=1+2 \eta_{i}-6 \epsilon_{i}, \quad r=16 \epsilon_{i}$,

respectively. Here the subscript $i$ means evaluation at the initial value of the inflaton, $\varphi_{i}$ i.e., at the horizon crossing. 
The number of e-foldings between $\varphi_{i}$ and $\varphi_{f}$ is given by

$N_{e}=\kappa^{2} \int_{\varphi_{f}}^{\varphi_{i}} d \varphi \frac{V}{V^{\prime}}$.

Another important observable is the amplitude of scalar perturbations given by

$A_{s}=\frac{\kappa^{4} V\left(\varphi_{i}\right)}{24 \pi^{2} \epsilon_{i}}$.

According to the PLANCK data (2018), the observed values of $n_{s}, r$, and $A_{s}$ are [44]

$$
n_{s}=0.9649 \pm 0.0042(68 \% \mathrm{CL}), \quad r<0.064(95 \% \mathrm{CL}),
$$

$$
\begin{aligned}
& \log \left(10^{10} A_{S}\right)=2.975 \pm 0.056(68 \% \mathrm{CL}) \\
& \Rightarrow A_{S} \approx 1.96 \times 10^{-9} .
\end{aligned}
$$

In our models, $n_{s}$ and $r$ depend only on $\alpha$ and $\operatorname{sgn}\left(\omega_{1}\right)$ (and not on the value of $\omega_{1}$ ) which determine the shape of the scalar potential. The observed value of $A_{s}\left(\sim 10^{-9}\right)$ can be used to fix the composite parameter $|\mu|^{2(\alpha-1)} / \omega_{1}^{\alpha-2}$ that is related to the inflaton mass via Eq. (48).

First, we numerically evaluate $n_{s}$ as a function of $\alpha$ for $N_{e}=50$ to 60 . The results of the evaluation are presented in Fig. 3. Figure 3a shows the tilt $n_{s}(\alpha)$ evaluated for a positive $\omega_{1}$ and $3<\alpha<\alpha_{*}$, while Fig. 3b shows the tilt $n_{s}(\alpha)$ evaluated for a negative $\omega_{1}$ and $3 \leq \alpha \leq 7.6$. The $\omega_{1}>0$ case, in part due to its limited domain of validity $(3<\alpha<$ $\left.\alpha_{*}\right)$, is fully compatible with the observations of the spectral tilt $n_{s}$. However, in the $\omega_{1}<0$ case, if $\alpha$ is greater than the certain value around 7.2 (let us call this value $\alpha_{\max }$ ), the predicted value of $n_{s}$ becomes smaller than the lower observational limit $n_{s}=0.9607 . .^{5}$ A more precise value of $\alpha_{\max }$ can be derived by finding $\varphi_{i}$ that solves the condition $n_{s}\left(\varphi_{i}\right)=0.9607$ and substituting this value in Eq. (54) to solve $N_{e}(\alpha)=60$. This results in

$\alpha_{\max } \approx 7.235$

Therefore, when $\omega_{1}<0$ we exclude the models with $\alpha>$ $\alpha_{\max }$.

As we show below, the tensor-to-scalar ratio $r$ decreases with increasing $\alpha$ and is always compatible with the limit $r<0.064$.

\footnotetext{
$\overline{5 \text { In fact, the } n_{s}}$ decreases quite rapidly after $\alpha_{\max }$. For example, already for $\alpha=10$ we have $n_{s} \approx 0.3614$.
}

4.1 The case $3 \leq \alpha \leq \alpha_{*}$ : starobinsky-like inflation

Let us divide our models into two classes for $3 \leq \alpha \leq \alpha_{*}$ and $\alpha_{*}<\alpha \leq \alpha_{\max }$, respectively. The reason is that in the range $3 \leq \alpha \leq \alpha_{*}$ the inflationary potential is truly Starobinskylike and has a single extremum, namely, the global minimum and the infinite plateau asymptotically approaching a constant positive height. In contrast, if $\alpha>\alpha_{*}$ the potential has a local maximum, which means that we get the hilltop inflationary models.

For simplicity, we restrict ourselves to integer $\alpha$, and proceed with calculating the inflationary parameters $n_{s}$ and $r$ for $3 \leq \alpha \leq \alpha_{*}$ by setting $N_{e}=55$. In this subsection, we take $\alpha=3,4,5,6$ ( $\alpha=3$ is the Starobinsky case) and, in addition, we include the upper limit $\alpha=\alpha_{*} \equiv(7+\sqrt{33}) / 2$. The results of our numerical calculations of $n_{s}$ and $r$ are in Table 1, and the corresponding scalar potentials for the chosen values of $\alpha$ are in Fig. 4.

The relation to the amplitude $A_{s}$ of CMB scalar perturbations in Eq. (55) is conveniently described by the composite parameter

$\Lambda^{6} \equiv \frac{|\mu|^{2(\alpha-1)}}{\left|\omega_{1}\right|^{\alpha-2}}$,

where $\Lambda$ has units of mass. When $\omega_{1}<0$ and $3 \leq \alpha \leq \alpha_{*}$, Eq. (57) yields $\Lambda \sim 10^{101 / 6} \mathrm{GeV} \sim 10^{16.8} \mathrm{GeV}$, whereas in the case of $\omega_{1}>0$ and $3<\alpha<\alpha_{*}$ we find

$\lim _{\alpha \rightarrow 3} \Lambda=0, \quad \lim _{\alpha \rightarrow \alpha_{*}} \Lambda=\infty$,

due to the behavior of $\gamma_{+}(\alpha)$ (see Eq. 32) in the scalar potential (51). Given $\alpha=4,5,6$, the parameter $\Lambda$ is of the order $10^{16.5}, 10^{16.8}, 10^{17.5} \mathrm{GeV}$, respectively.

The inflaton mass is $m_{\varphi} \sim 10^{13} \mathrm{GeV}$ irrespectively of the choice of $\alpha$ and $\operatorname{sgn}\left(\omega_{1}\right)$.

\subsection{The case $\alpha>\alpha_{*}$ : hilltop inflation}

The viable hilltop inflationary models are limited to $\alpha_{*}<$ $\alpha \leq \alpha_{\max }$ with $\alpha_{*}=(7+\sqrt{33}) / 2 \approx 6.372$ and $\alpha_{\max } \approx$ 7.235. Let us consider $\alpha=7$, because it is the only integer between $\alpha_{*}$ and $\alpha_{\max }$.

Taking $N_{e}=60$ (for a better fit of $n_{s}$ with PLANCK data), we calculate the parameters as follows: $n_{s} \approx 0.9635$, $r \approx 0.0002$, and $\Lambda \sim 10^{16.8} \mathrm{GeV}$. The form of the scalar potential is given in Fig. 5 where the local maximum $\varphi_{+}$and the starting point of inflation $\varphi_{i}$ are shown.

\subsection{SUSY breaking scale}

Let us parametrize the SUSY breaking scale by the gravitino mass that can be read off from Fig. 2 after taking into 
Fig. 3 The tilt $n_{s}$ as a function of $\alpha$ for positive and negative $\omega_{1}$, and $50 \leq N_{e} \leq 60$. The values $n_{s}=0.9691$ and $n_{s}=0.9607$ are the upper and lower observational limits (68\%CL), respectively
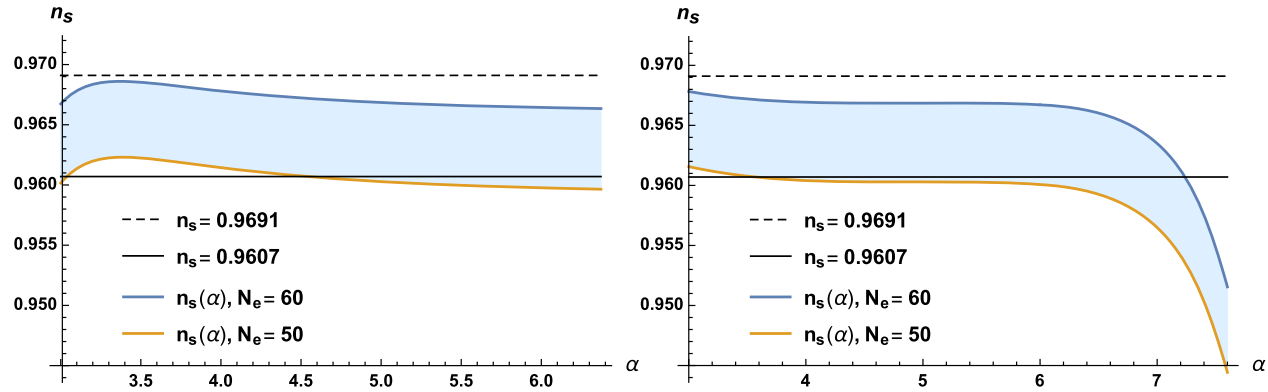

(a) $\omega_{1}>0$

(b) $\omega_{1}<0$

Table 1 The predictions for the inflationary parameters $\left(n_{s}, r\right)$, and the values of $\varphi$ at the horizon crossing $\left(\varphi_{i}\right)$ and at the end of inflation $\left(\varphi_{f}\right)$, in the case $3 \leq \alpha \leq \alpha_{*}$ with both signs of $\omega_{1}$. The $\alpha$ parameter is taken to be integer, except of the upper limit $\alpha_{*} \equiv(7+\sqrt{33}) / 2$

\begin{tabular}{lllllll}
\hline$\alpha$ & 3 & 4 & & 5 & 6 & $\alpha_{*}$ \\
\hline $\operatorname{sgn}\left(\omega_{1}\right)$ & - & + & - & $+/-$ & + & - \\
$n_{s}$ & 0.9650 & 0.9649 & 0.9640 & 0.9639 & 0.9634 & 0.9637 \\
$r$ & 0.0035 & 0.0010 & 0.0013 & 0.0007 & 0.0005 & 0.0004 \\
$-\kappa \varphi_{i}$ & 5.3529 & 3.5542 & 3.9899 & 3.2657 & 3.0215 & 2.7427 \\
$-\kappa \varphi_{f}$ & 0.9402 & 0.7426 & 0.8067 & 0.7163 & 0.6935 & 0.0032 \\
\hline
\end{tabular}

Fig. 4 The scalar potentials for both signs of $\omega_{1}$ and the integer values of $\alpha$ in the range $3 \leq \alpha \leq \alpha_{*}$. The inflaton mass (see Eq. 48) and $\kappa$ are set to be one

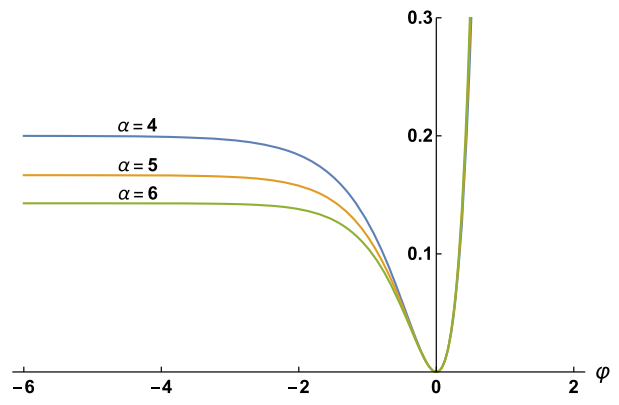

(a) $\omega_{1}>0$

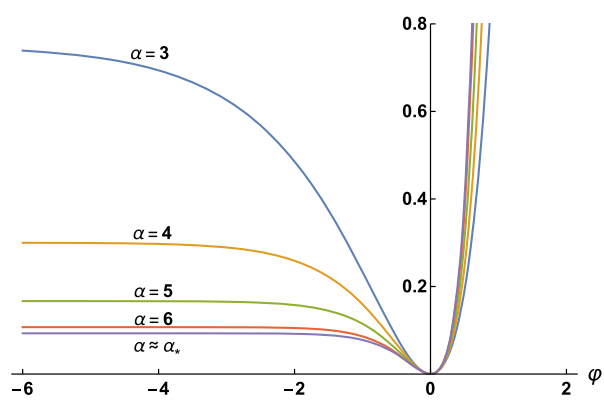

(b) $\omega_{1}<0$

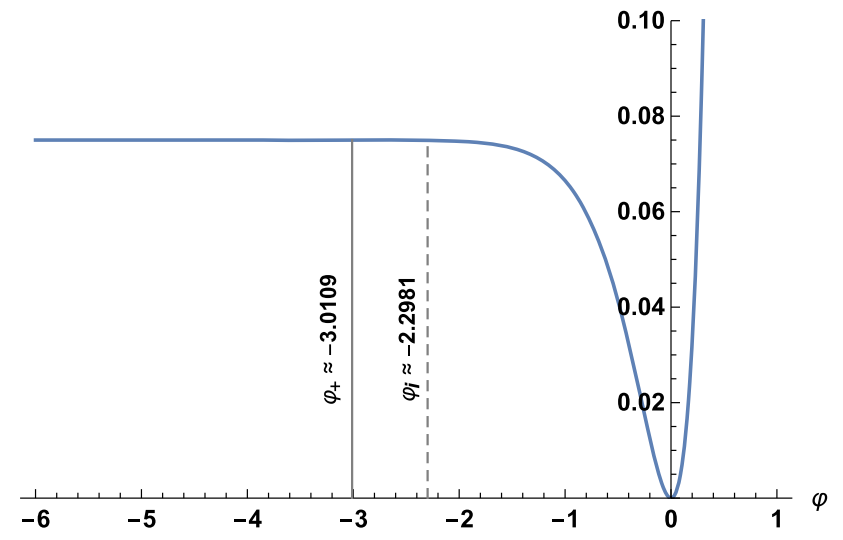

Fig. 5 The scalar potential (51) for $\alpha=7, \omega_{1}<0$, and thus $\gamma=\gamma_{-}$ $\left(m_{\varphi}=\kappa=1\right)$. The solid vertical line shows the local maximum $\varphi_{+}$, and the dashed vertical line shows the starting point of inflation $\varphi_{i}$ when $N_{e}=60$

account the inflaton mass fixed by the observed value of $A_{s}$ in Eq. (57). For example, if $\omega_{1}>0, m_{3 / 2}$ ranges from the inflationary scale to arbitrarily high scale (as $\alpha \rightarrow 3$ or $\alpha \rightarrow \alpha_{*}$ ).
If $\omega_{1}<0$ and $3 \leq \alpha \leq \alpha_{\max }$, the gravitino mass is always lower than $m_{\varphi}$ by at most one order of the magnitude. The exception is the value $\alpha=3$ when $m_{3 / 2} \geq m_{\varphi} / 2$ as is shown in Eq. (26).

In Table 2 we provide the explicit values of $m_{\varphi}, m_{t^{\prime}}$, $m_{3 / 2},\left\langle F_{T}\right\rangle$, and $\langle D\rangle$ for the integer values of $\alpha$ between 3 and $\alpha_{\max } \approx 7.235$, derived from our models by fixing $A_{s}$ according to Eq. (57). This fixes $\langle D\rangle=\kappa^{-2} g \xi$ [by using Eqs. (29) and (32)], but it is not enough to fix $\left\langle F_{T}\right\rangle$ in the $\omega_{1}>0$ case (when $\omega_{1}<0$, the $\left\langle F_{T}\right\rangle$ identically vanishes except for $\alpha=3$ where it is undetermined). In particular, for $\alpha=4,5,6$, Eq. (42) yields

$\left\langle F_{T}\right\rangle=\frac{5}{4} \bar{\mu} \kappa, \quad\left\langle F_{T}\right\rangle=\sqrt{\frac{3}{5}} \frac{\bar{\mu}|\mu|}{\sqrt{\omega_{1}}} \kappa, \quad\left\langle F_{T}\right\rangle=\frac{7 \bar{\mu}|\mu|^{2}}{162 \omega_{1}} \kappa$,

respectively. There is always enough freedom to choose the values of $\left\langle F_{T}\right\rangle$ independently of the parameter $\Lambda^{6}=$ 
Table 2 The masses of inflaton, axion and gravitino, and the VEVs of $F$ - and $D$-fields derived from our models by fixing the amplitude $A_{s}$ according to PLANCK data - see Eq. (57). The value of $\left\langle F_{T}\right\rangle$ for a positive $\omega_{1}$ is not fixed by $A_{s}$

$\left.\begin{array}{|c|c|c|c|c|c|c|c|c|}\hline \alpha & 3 & \multicolumn{2}{|c|}{4} & \multicolumn{2}{|c|}{5} & \multicolumn{2}{|c|}{6} & 7 \\ \hline \operatorname{sgn}\left(\omega_{1}\right) & - & + & - & + & - & + & - & - \\ \hline m_{\varphi} & 2.83 & 2.95 & 2.73 & 2.71 & 2.71 & 2.53 & 2.58 & 1.86 \\ \hline m_{t^{\prime}} & 0 & 0.93 & 1.73 & 2.02 & 2.02 & 4.97 & 2.01 & 1.56 \\ \hline m_{3 / 2} & \geq 1.41 & 2.80 & 0.86 & 2.56 & 0.64 & 3.91 & 0.49 & 0.29\end{array}\right\} \times 10^{13} \mathrm{GeV}$

$|\mu|^{2(\alpha-1)} /\left|\omega_{1}\right|^{\alpha-2}$ that is fixed by the observed amplitude $A_{s}$.

The most important prediction of our models (apart from the existence of the upper limit $\alpha_{\max }$ ) for integer $\alpha$ is the very high SUSY breaking scale parametrized by the superheavy gravitino mass $m_{3 / 2}$ of the order of $10^{12}$ to $10^{13} \mathrm{GeV}$. For fractional $\alpha$, if $\omega_{1}>0$, the SUSY breaking scale can be arbitrarily high as $\alpha$ approaches 3 or $\alpha_{*}$.

\section{Conclusion}

In this paper we studied a class of unified models of inflation, spontaneous SUSY breaking, and dark energy (described by the positive cosmological constant) based on the generalized dilaton-axion multiplet coupled to $N=1$ supergravity with the Kähler potential and superpotential

$K=-\alpha \log (T+\bar{T}), \quad W=\lambda+\mu T$,

in the presence of a single vector multiplet with the gauge kinetic function $f=1$. In order to uplift the resulting AdS vacuum, we used the alternative FI term introduced in Refs. $[14,15]$. This allowed us to get a tunable positive cosmological constant and the D-term contribution to SUSY breaking.

We showed that, unless $\alpha \geq 3$, the scalar potential is unstable. The choice $\alpha=3$ leads to the Starobinsky potential for the dilaton $\varphi$, while the axion direction is flat, i.e. the axion mass has to be generated by quantum corrections. On the other hand, for $\alpha>3$ the axion has a positive non-vanishing mass squared and is automatically stabilized. Once the axion acquires a VEV, those models lead to the effective singlefield inflation where inflaton is identified with dilaton. We found that the shape of the potential, and thus the inflationary observables $n_{s}$ and $r$, are controlled by $\alpha$ and the sign of the real parameter $\omega_{1} \equiv \bar{\lambda} \mu+\lambda \bar{\mu}$, whereas the amplitude of scalar perturbations is related to the value of the composite parameter $\Lambda^{6}=|\mu|^{2(\alpha-1)} /\left|\omega_{1}\right|^{\alpha-2}$. In particular, when
$3 \leq \alpha \leq \alpha_{*}\left(\alpha_{*} \approx 6.372\right)$, the derived inflation is of the Starobinsky type where the inflaton rolls down an infinite plateau, while for $\alpha>\alpha_{*}$ the potential has a local maximum (hilltop).

One of our main results is the upper limit on $\alpha$ : by analyzing the dependence of $n_{s}$ on $\alpha$ (Fig. 3), we found that $\alpha_{\max } \approx 7.235$ is the maximum value that can reproduce the observed spectral tilt $n_{s}=0.9649 \pm 0.0042$. More precise observations of $n_{s}$ may further reduce the value of $\alpha_{\max }$.

Another important prediction of our models is the (very) high-scale SUSY breaking, so that for integer $\alpha$ the gravitino mass is roughly of the order of the inflaton mass, $m_{3 / 2} \sim m_{\varphi} \sim 10^{13} \mathrm{GeV}$ (for fractional $\alpha, m_{3 / 2}$ can be arbitrarily high). In comparison, the scale of the D-term is $\sqrt{|\langle D\rangle|}=\kappa^{-1} \sqrt{g|\xi|} \sim 10^{15.5} \mathrm{GeV}$. We explicitly derived the masses of dilaton, axion and gravitino, together with the SUSY breaking parameters $\left\langle F_{T}\right\rangle$ and $\langle D\rangle$ for $\alpha=$ 3, 4, 5, 6, 7 (see Table 2). It is interesting that the models with a negative $\omega_{1}$ have the vanishing F-terms $\left\langle F_{T}\right\rangle=0$ (except for $\alpha=3$ ), so that SUSY is broken purely by the Dterm. Those models may be interesting in connection to the universality of scalar masses in the Supersymmetric Standard Model due to the vanishing F-terms, see e.g., Refs. [45,46], though more research is needed in this direction. The axions and gravitinos in our models can be used as the superheavy dark matter along the lines of Refs. [11,47-51].

Although the origin of the alternative FI terms in superstring theory is not clear, the generalized dilaton-axion superfield with the Kähler potential given by Eq. (62) with $\alpha=1,2, \ldots, 7$ may be derived from M-theory compactified on a $G_{2}$ manifold [52-54], where the effective $N=1$, $D=4$ supergravity has seven complex scalars parametrizing the $S L(2 ; \mathbb{R})^{7} / S O(2)^{7}$ manifold with the Kähler potential

$K=-\sum_{i=1}^{7} \log \left(\Phi_{i}+\bar{\Phi}_{i}\right)$.

Then various integer values of $\alpha$ can be obtained by selecting a desired number of $\Phi_{i}$ superfields and setting the others to 
be constants. For example, in order to obtain $\alpha=5$, we can choose $\Phi_{1}=\Phi_{2}=\cdots=\Phi_{5}$ and $\Phi_{6}=\Phi_{7}=$ const.

Acknowledgements Y.A. and A.C. were supported by the CUniverse research promotion project of Chulalongkorn University under the grant reference CUAASC. Y.A. was also supported by the Ministry of Education and Science of the Republic of Kazakhstan under the grant reference BR05236322. S.V.K. was supported by the World Premier International Research Center Initiative (WPI), MEXT, Japan, and the Competitiveness Enhancement Program of Tomsk Polytechnic University in Russia.

Data Availability Statement This manuscript has associated data in a data repository. [Authors' comment: All of the data used in this paper are available in Refs. [44].]

Open Access This article is distributed under the terms of the Creative Commons Attribution 4.0 International License (http://creativecomm ons.org/licenses/by/4.0/), which permits unrestricted use, distribution, and reproduction in any medium, provided you give appropriate credit to the original author(s) and the source, provide a link to the Creative Commons license, and indicate if changes were made. Funded by SCOAP ${ }^{3}$.

\section{Appendix: $N=1$ supergravity and the alternative FI terms}

The bosonic sector of the standard four-dimensional $N=1$ matter-coupled supergravity reads (in Planck units, $\kappa=1)^{6}$

$$
\begin{aligned}
e^{-1} \mathcal{L}= & \frac{1}{2} R-K_{i \bar{j}} D_{m} \Phi^{i} \bar{D}^{m} \Phi^{j}-\frac{1}{4} f_{A B}^{R} F_{m n}^{A} F^{B, m n} \\
& -\frac{i}{4} f_{A B}^{I} \tilde{F}_{m n}^{A} F^{B, m n}-V_{F}-V_{D},
\end{aligned}
$$

whose the F- and D-type scalar potentials are given by

$$
\begin{aligned}
V_{F} & =e^{K}\left[K^{i \bar{j}}\left(W_{i}+K_{i} W\right)\left(\bar{W}_{\bar{j}}+K_{\bar{j}} \bar{W}\right)-3|W|^{2}\right], \\
V_{D} & =\frac{g^{2}}{2} f_{R}^{A B} \mathscr{D}_{A} \mathscr{D}_{B},
\end{aligned}
$$

where $K=K\left(\Phi_{i}, \bar{\Phi}_{i}\right)$ is the Kähler potential depending upon the chiral (complex) scalar fields $\Phi_{i}, F_{m n}^{A}=$ $\partial_{m} A_{n}^{A}-\partial_{n} A_{m}^{A}+g f^{A B C} A_{m}^{B} A_{n}^{C}$ is the field strength of the vector fields $A_{m}^{A}, W=W\left(\Phi_{i}\right)$ is the holomorphic superpotential, $f=f\left(\Phi_{i}\right)$ is the holomorphic gauge kinetic function with $f_{R} \equiv \operatorname{Re} f, g$ is the gauge coupling, and $\mathscr{D}_{A}$ are the Killing potentials (the moment maps) of a given gauge group. We use the notation $K^{i \bar{j}} \equiv K_{i \bar{j}}^{-1}$, where $K_{i \bar{j}} \equiv \frac{\partial^{2} K}{\partial \Phi_{i} \partial \bar{\Phi}_{j}}$, $W_{i} \equiv \frac{\partial W}{\partial \Phi_{i}}$, and $f^{A B} \equiv f_{A B}^{-1}$ with $A, B$ as the gauge group indices. The covariant derivatives of the chiral fields are

$D_{m} \Phi^{i}=\partial_{m} \Phi^{i}-g A_{m}^{A} X_{A}^{i}$,

where $X_{A}^{i}$ are the Killing vectors of the gauge symmetries.

\footnotetext{
${ }^{6}$ A derivation of this action from curved superspace can be found e.g., in Ref. [55].
}

The potentials (65) and (66), as well as the full Lagrangian of $N=1$ supergravity, are invariant with respect to the Kähler-Weyl transformations

$$
K \rightarrow K+\Sigma+\bar{\Sigma}, \quad W \rightarrow W e^{-\Sigma},
$$

where $\Sigma$ is an arbitrary chiral (super)field.

In Refs. [14,15] the alternative Fayet-Iliopoulos-type terms in the case of an abelian gauge group were introduced, which do not require gauging the R-symmetry. The FI term with the simplest contribution of the bosonic terms (when using the superspace approach of Ref. [55]) reads

$$
\mathcal{L}_{\mathrm{FI}}=-g \xi \int d^{2} \Theta 2 \mathcal{E} \overline{\mathcal{P}}\left(\frac{\mathcal{W}^{2} \overline{\mathcal{W}}^{2}}{\mathcal{P} \mathcal{W}^{2} \overline{\mathcal{P}}^{2}} \mathcal{D} \mathcal{W}\right)+\text { h.c. }
$$

where $\xi$ is the real FI constant, $g$ is the $U(1)$ gauge coupling, and $\mathcal{P}$ is the chiral projector $\mathcal{P} \equiv \mathcal{D}^{2}-8 \overline{\mathcal{R}}, \overline{\mathcal{P}} \equiv \overline{\mathcal{D}}^{2}-8 \mathcal{R}$; the (chiral) superfield strength of the vector superfield $\mathcal{V}$ is defined by $\mathcal{W}_{\alpha} \equiv-\frac{1}{4} \overline{\mathcal{P}} \mathcal{D}_{\alpha} \mathcal{V}$ with $\mathcal{W}^{2} \equiv \mathcal{W}^{\alpha} \mathcal{W}_{\alpha}$. Then the scalar bosonic part of the FI term (69) is just $\mathcal{L}_{\mathrm{FI}}=$ $-e g \xi D$. In order to couple the new FI term to chiral superfields in a Kähler-Weyl-invariant manner, one must rescale $\xi \rightarrow e^{-K / 3} \xi[13,42]$.

Our models in this paper are described by the potentials

$K=-\alpha \log (T+\bar{T})$,

$W=\lambda+\mu T, \quad f=1$,

with complex parameters $\lambda$ and $\mu$. The shift-symmetry $T \rightarrow$ $T+i a$ is not gauged, the Killing potential vanishes, while $V_{D}$ includes the constant FI contribution (absent in the standard supergravity) that we use for a dS uplift. After using Eq. (70) and the parametrization $T=e^{-\sqrt{\frac{2}{\alpha}} \phi}+i t$, the full bosonic Lagrangian accounting for the FI contribution takes the form

$$
\begin{aligned}
& e^{-1} \mathcal{L}=\frac{1}{2} R-\frac{1}{2} \partial_{m} \phi \partial^{m} \phi-\frac{\alpha}{4} e^{\sqrt{\frac{8}{\alpha}} \phi} \partial_{m} t \partial^{m} t \\
& -\frac{1}{4} F_{m n} F^{m n}-V_{F}-V_{D},
\end{aligned}
$$

where $V_{F}$ and $V_{D}$ are given by Eqs. (2) and (8), respectively.

\section{References}

1. H.P. Nilles, Supersymmetry, supergravity and particle physics. Phys. Rep. 110, 1-162 (1984)

2. I.J.R. Aitchison, Supersymmetry in Particle Physics. An Elementary Introduction (Cambridge University Press, Cambridge, 2007)

3. S.V. Ketov, Supergravity and early universe: the meeting point of cosmology and high-energy physics. Int. J. Mod. Phys. A 28, 1330021 (2013). arXiv:1201.2239 [hep-th]

4. S.V. Ketov, MYu. Khlopov, Cosmological probes of supersymmetric field theory models at superhigh energy scales. Symmetry 11(4), 511 (2019) 
5. F. Farakos, A. Kehagias, A. Riotto, On the Starobinsky model of inflation from supergravity. Nucl. Phys. B 876, 187-200 (2013). arXiv:1307.1137 [hep-th]

6. S. Ferrara, R. Kallosh, A. Linde, M. Porrati, Higher order corrections in minimal supergravity models of inflation. JCAP 1311, 046 (2013). arXiv:1309.1085 [hep-th]

7. S.V. Ketov, T. Terada, Generic scalar potentials for inflation in supergravity with a single chiral superfield. JHEP 12, 062 (2014). arXiv: 1408.6524 [hep-th]

8. S.V. Ketov, T. Terada, Inflation in supergravity with a single chiral superfield. Phys. Lett. B 736, 272-277 (2014). arXiv:1406.0252 [hep-th]

9. Y. Aldabergenov, S.V. Ketov, SUSY breaking after inflation in supergravity with inflaton in a massive vector supermultiplet. Phys. Lett. B 761, 115-118 (2016). arXiv:1607.05366 [hep-th]

10. Y. Aldabergenov, S.V. Ketov, Higgs mechanism and cosmological constant in $N=1$ supergravity with inflaton in a vector multiplet. Eur. Phys. J. C 77(4), 233 (2017). arXiv:1701.08240 [hep-th]

11. A. Addazi, S.V. Ketov, MYu. Khlopov, Gravitino and Polonyi production in supergravity. Eur. Phys. J. C 78(8), 642 (2018). arXiv:1708.05393 [hep-ph]

12. Y. Aldabergenov, S.V. Ketov, Removing instability of inflation in Polonyi-Starobinsky supergravity by adding FI term. Mod. Phys. Lett. A 91(05), 1850032 (2018). arXiv:1711.06789 [hep-th]

13. Y. Aldabergenov, S.V. Ketov, R. Knoops, General couplings of a vector multiplet in $N=1$ supergravity with new FI terms. Phys. Lett. B 785, 284-287 (2018). arXiv:1806.04290 [hep-th]

14. N. Cribiori, F. Farakos, M. Tournoy, A. van Proeyen, FayetIliopoulos terms in supergravity without gauged R-symmetry. JHEP 04, 032 (2018). arXiv:1712.08601 [hep-th]

15. S.M. Kuzenko, Taking a vector supermultiplet apart: alternative Fayet-Iliopoulos-type terms. Phys. Lett. B 781, 723-727 (2018). arXiv:1801.04794 [hep-th]

16. H. Abe, Y. Aldabergenov, S. Aoki, S.V. Ketov, Massive vector multiplet with Dirac-Born-Infeld and new Fayet-Iliopoulos terms in supergravity. JHEP 09, 094 (2018). arXiv:1808.00669 [hep-th]

17. H. Abe, Y. Aldabergenov, S. Aoki, S.V. Ketov, PolonyiStarobinsky supergravity with inflaton in a massive vector multiplet with DBI and FI terms. Class. Quant. Gravit. 36(7), 075012 (2019). arXiv:1812.01297 [hep-th]

18. J. Polonyi, Generalization of the massive scalar multiplet coupling to the supergravity. Hungary Central Inst. Res. KFKI-77-93 (1977, REC. JUL 1978), p. 5 (unpublished)

19. J.R. Ellis, C. Kounnas, D.V. Nanopoulos, Phenomenological SU(1,1) supergravity. Nucl. Phys. B 241, 406-428 (1984)

20. E. Cremmer, S. Ferrara, C. Kounnas, D.V. Nanopoulos, Naturally vanishing cosmological constant in $\mathrm{N}=1$ supergravity. Phys. Lett. 133B, 61 (1983)

21. J.R. Ellis, A.B. Lahanas, D.V. Nanopoulos, K. Tamvakis, No-scale supersymmetric standard model. Phys. Lett. 134B, 429 (1984)

22. J.R. Ellis, C. Kounnas, D.V. Nanopoulos, No scale supersymmetric guts. Nucl. Phys. B 247, 373-395 (1984)

23. S. Cecotti, Higher derivative supergravity is equivalent to standard supergravity coupled to matter. 1. Phys. Lett. B 190, 86-92 (1987)

24. S.J. Gates Jr., S.V. Ketov, Superstring-inspired supergravity as the universal source of inflation and quintessence. Phys. Lett. B 674, 59-63 (2009). arXiv:0901.2467 [hep-th]

25. S. Ketov, $F(R)$ supergravity. AIP Conf. Proc. 1241(1), 613-619 (2010). arXiv:0910.1165 [hep-th]

26. A.A. Starobinsky, A new type of isotropic cosmological models without singularity. Phys. Lett. B 91, 99-102 (1980). [771(1980)]

27. J. Ellis, M.A.G. Garcia, D.V. Nanopoulos, K.A. Olive, Phenomenological aspects of no-scale inflation models. JCAP 1510(10), 003 (2015). arXiv:1503.08867 [hep-ph]
28. J. Ellis, M.A.G. Garcia, D.V. Nanopoulos, K.A. Olive, Noscale inflation. Class. Quant. Gravit. 33(9), 094001 (2016). arXiv:1507.02308 [hep-ph]

29. J. Ellis, D.V. Nanopoulos, K.A. Olive, From $R^{2}$ gravity to no-scale supergravity. Phys. Rev. D 97(4), 043530 (2018). arXiv:1711.11051 [hep-th]

30. J. Ellis, B. Nagaraj, D.V. Nanopoulos, K.A. Olive, De Sitter vacua in no-scale supergravity. JHEP 11, 110 (2018). arXiv:1809.10114 [hep-th]

31. R. Kallosh, A. Linde, D. Roest, Superconformal inflationary $\alpha$ attractors. JHEP 11, 198 (2013). arXiv:1311.0472 [hep-th]

32. D. Roest, M. Scalisi, Cosmological attractors from $\alpha$-scale supergravity. Phys. Rev. D 92, 043525 (2015). arXiv:1503.07909 [hepth]

33. A. Linde, Single-field $\alpha$-attractors. JCAP 1505, 003 (2015). arXiv: 1504.00663 [hep-th]

34. J. Ellis, D.V. Nanopoulos, K.A. Olive, S. Verner, A general classification of Starobinsky-like inflationary avatars of $\mathrm{SU}(2,1) / \mathrm{SU}(2) \times \mathrm{U}(1)$ no-scale supergravity. JHEP 03, 099 (2019). arXiv:1812.02192 [hep-th]

35. J. Ellis, D.V. Nanopoulos, K.A. Olive, S. Verner, Unified No-Scale Attractors. arXiv:1906.10176 [hep-th]

36. S. Ferrara, R. Kallosh, A. Linde, M. Porrati, Minimal supergravity models of inflation. Phys. Rev. D 88(8), 085038 (2013). arXiv:1307.7696 [hep-th]

37. I. Antoniadis, A. Chatrabhuti, H. Isono, R. Knoops, Inflation from supergravity with gauged R-symmetry in de Sitter vacuum. Eur. Phys. J. C 76(12), 680 (2016). arXiv:1608.02121 [hep-ph]

38. F. Farakos, A. Kehagias, A. Riotto, Liberated $\mathcal{N}=1$ supergravity. JHEP 06, 011 (2018). arXiv:1805.01877 [hep-th]

39. I. Antoniadis, A. Chatrabhuti, H. Isono, R. Knoops, The cosmological constant in supergravity. Eur. Phys. J. C 78(9), 718 (2018). arXiv: 1805.00852 [hep-th]

40. N. Cribiori, F. Farakos, M. Tournoy, Supersymmetric Born-Infeld actions and new Fayet-Iliopoulos terms. JHEP 03, 050 (2019). arXiv:1811.08424 [hep-th]

41. I. Antoniadis, J.-P. Derendinger, F. Farakos, G. TartaglinoMazzucchelli, New Fayet-Iliopoulos terms in $\mathcal{N}=2$ supergravity. arXiv: 1905.09125 [hep-th]

42. I. Antoniadis, A. Chatrabhuti, H. Isono, R. Knoops, FayetIliopoulos terms in supergravity and D-term inflation. Eur. Phys. J. C 78(5), 366 (2018). arXiv:1803.03817 [hep-th]

43. Y. Aldabergenov, No-scale supergravity with new Fayet-Iliopoulos term. Phys. Lett. B 795, 366-370 (2019). arXiv:1903.11829 [hepth]

44. Planck Collaboration, Y. Akrami et al., Planck 2018 Results. X. Constraints on Inflation. arXiv:1807.06211 [astro-ph.CO]

45. G.R. Dvali, A. Pomarol, Supersymmetry breaking with vanishing F terms in supergravity theories. Phys. Lett. B 410, 160-166 (1997). arXiv:hep-ph/9706429 [hep-ph]

46. E. Dudas, S.K. Vempati, Large D-terms, hierarchical soft spectra and moduli stabilisation. Nucl. Phys. B 727, 139-162 (2005). arXiv:hep-th/0506172 [hep-th]

47. A. Addazi, A. Marciano, S.V. Ketov, MYu. Khlopov, Physics of superheavy dark matter in supergravity. Int. J. Mod. Phys. D 27(06), 1841011 (2018)

48. V. Kuzmin, I. Tkachev, Matter creation via vacuum fluctuations in the early universe and observed ultrahigh-energy cosmic ray events. Phys. Rev. D 59, 123006 (1999). arXiv:hep-ph/9809547 [hep-ph]

49. D.J.H. Chung, E.W. Kolb, A. Riotto, Nonthermal supermassive dark matter. Phys. Rev. Lett. 81, 4048-4051 (1998). arXiv:hep-ph/9805473 [hep-ph]

50. D.J.H. Chung, E.W. Kolb, A. Riotto, Superheavy dark matter. Phys. Rev. D 59, 023501 (1999). arXiv:hep-ph/9802238 [hep-ph] 
51. D.J.H. Chung, E.W. Kolb, A. Riotto, Production of massive particles during reheating. Phys. Rev. D 60, 063504 (1999). arXiv:hep-ph/9809453 [hep-ph]

52. M.J. Duff, S. Ferrara, Generalized mirror symmetry and trace anomalies. Class. Quant. Gravit. 28, 065005 (2011). arXiv:1009.4439 [hep-th]

53. M.J. Duff, S. Ferrara, Four curious supergravities. Phys. Rev. D 83, 046007 (2011). arXiv:1010.3173 [hep-th]
54. S. Ferrara, R. Kallosh, Seven-disk manifold, $\alpha$-attractors, and $B$ modes. Phys. Rev. D 94(12), 126015 (2016). arXiv:1610.04163 [hep-th]

55. J. Wess, J. Bagger, Supersymmetry and supergravity (Princeton University Press, Princeton, 1992) 